$$
2\left(1-e^{2}\right)^{3 / 2} \sum_{n=1}^{\infty}(-1)^{n}(n+1)\left(\frac{e}{2}\right)^{n} \sigma^{\prime}
$$

where, $\sigma^{\prime}=\left(\begin{array}{c}n-1 \\ \frac{n-1}{2}\end{array}\right)$, for $n$ odd

$$
=0 \text {, for } n \text { even. }
$$

Extracting $\left(\frac{e}{2}\right)$ from equation (6) we obtain the expression :

$$
e\left(1-e^{2}\right)^{3 / 2} \sum_{n=1}^{\infty}(-1)^{n}(n+1)\left(\frac{e}{2}\right)^{n-1} \sigma^{\prime}
$$

But,

$$
\sum_{n=1}^{\infty}(-1)^{n}(n+1)\left(\frac{e}{2}\right)^{n-1} \sigma^{\prime}=-2\left(1-e^{2}\right)^{-8 / 2}
$$

Hence, the coefficient of $\sin \nu$ is $-2 e$ for all $n$, and the solution (5) may be expressed as :

$$
\begin{aligned}
M=\nu-2 e \sin \nu+2\left(1-e^{2}\right)^{3} \cdot 2 & \sum_{n=2}^{\infty} \sum_{\lambda=0}^{\left[\begin{array}{l}
n \\
2
\end{array}\right]}(-1)^{n}(n+1) \\
& \left(\frac{e}{2}\right)^{n}\left(\frac{n}{\lambda}\right) \frac{\sin (n-2 \lambda) \nu}{n-2 \lambda}
\end{aligned}
$$

where $n \neq 2 \lambda+1$.

As from equation (2), we obtain as far as terms in $e^{4}$, the usual expression :

$$
\begin{array}{r}
M=\nu-2 e \sin \nu+\left(\frac{3 e^{2}}{4}+\frac{e^{4}}{8}\right) \sin 2 \nu-\frac{e^{3}}{3} \sin 3 \nu+ \\
\frac{5 e^{4}}{32} \sin 4 \nu
\end{array}
$$

Department of Mathematics,

\section{J. BRookes}

Collyge of Advanced Technology,

Costa Groen, Birmingham, 4.

${ }^{1}$ Moulton, F. A., An Introduction to Celestial Mechanics, 164, 172 (Macmillan, 1959).

" Brown, E. W., An Introductory Treatise on the Lunar Theory, 31 (Cambridge Univ. Press, 1896).

"Smart, W. M., Celestial Mechanics, 38 (Longmans, 1953).

+ smart, W. M., Celestial Mechanics, 32 (Longmans, 1953).

'Brown, E. W., and Shook, C. A., Planetary Theory, 37 (Cambridge Univ. Press, 1933).

\section{The Complete n-Point Graph}

A COMPLETE $n$-point graph consists of $n$ points in a plane (or on the surface of a sphere), each joined to all the others by lines, not necessarily straight. The problem of determining the minimum number of intersections, $m$, for various values of $n$ is being investigated.

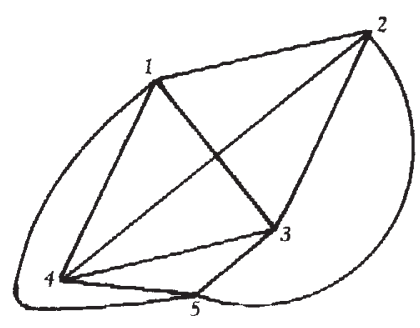

Fig. 1
Fig. 1 shows a complete 5-graph which has 1 intersection, this being the minimum for $n=5$.

The problem does not appear tractable analytically, so it was programmed for the University of London Mercury computer. The programme was written on the assumption that a minimum for $n+1$ points can be obtained by adding an extra point in a suitable place on a minimum solution for $n$ points. However, the computations have proved that this apparently natural assumption is false : two different minimum configuratiens for $n=7, m=9$ were taken and one led to the true minimum $n=8, m=18$, while the other led to $n=8, m=19$.

I thank Dr. A. D. Booth for help and encouragement.

Department of Numerical Automation,

H. P. Goodman

Birkbeck College

(University of London),

Malet Streot, W.C.I.

\section{STATISTICS}

\section{A Basic Notion in the Theory of Cognitive Systems}

IN a previous communication ${ }^{1}$ a network was described which would resolve linear relations among continuous input signals. It was indicated that for times greater than the resolution time (for some intrinsic error criterion) this net could follow changing relations and thus, by means of other nets, one could observe the relations between relations and so on. The process described is essentially that of ascending a scale of logical types. To characterize this more generally, consider a binary time series $y(t)$ of discrete time $t$. If this contains $2^{r} \leqslant 2^{m}$ structures of length it can be described by a logical function :

$$
y(t+1)=f\left\{y(t) \ldots y(t-m), x_{1}(t) \ldots x_{r}(t)\right\}
$$

where it is supposed that the variance $\mu(x)$ has been minimized. The $x$ 's then describe, roughly speaking, which of the $2^{r}$ structures is under consideration. But this by no means determines the function uniquely; there is an enormous redundancy of something like $2^{2^{r}}\left(2^{m} / m\right.$ ) possibilities (as examination of the periodic case, $r=0$, shows). However, for realization of logical functions it is well known that there are nets which are minimal with respect to number $v(f)$ of connoxions required. In this case one may expect to obtain effective unicity by requiring the minimiza. tion of a function such as :

$$
H(f, x)=\log v(f)+\log \mu(x)
$$

If $H(f, x)_{\min .}<\log \mu(y)$ we say that $(1)$ is a resolving transformation $f^{(1)}$ of type 1 , written formally as :

$$
y=f^{*} x^{(1)}
$$

Clearly the process could be repeated on the variables $x$ to obtain higher types, but this requires a notion, in a cortain sense dual to resolving transformation, which I call selective punctuation, $y^{(1)}$. This is the insertion of a sequence $y^{(1)}$ of length $m$ into the signal and may be regarded as the formalization of an experiment to test whether the $x^{(1)}$ so generated by the natural continuation of $y^{(1)}$ can be resolved into an $f^{(2)}$, and so on.

M. C. Goodall

Department of Biology,

Massachusetts Institute of Technology.

' Goodall, M. C., Nature, 185, 657 (1960). 